\title{
CORRECTION
}

\section{Correction to: A role for tetraspanin proteins in regulating fusion induced by Burkholderia thailandensis}

\author{
Atiga Elgawidi ${ }^{1}$ Muslim Idan Mohsin ${ }^{1,3} \cdot{\text { Fawwaz } \text { Ali }^{1,4} \cdot \text { Amyleigh Watts }^{1} \cdot \text { Peter N. Monk }}^{2} \cdot$ Mark S. Thomas ${ }^{2}$. \\ Lynda J. Partridge ${ }^{1}$
}

Published online: 3 June 2020

(c) Springer-Verlag GmbH Germany, part of Springer Nature 2020

\section{Correction to: Medical Microbiology and Immunology https://doi.org/10.1007/s00430-020-00670-6}

The original article has been corrected.

In the original article, incorrect Figures were published with incorrect captions. The correct Figs. 1, 2, 3, 4, 5, 6, 7 and 8 and captions are given below.

The original article can be found online at https://doi.org/10.1007/ s00430-020-00670-6.

Lynda J. Partridge

1.partridge@sheffield.ac.uk

1 Department of Molecular Biology and Biotechnology, University of Sheffield, Sheffield S10 2TN, UK

2 Department of Infection, Immunity and Cardiovascular Disease, Medical School, University of Sheffield, Sheffield S10 2RX, UK

3 Present Address: Department of Pathological Analyses, University of Kufa, Kufa, Iraq

4 Present Address: Mosul Technical Institute, Northern Technical University, Mosul, Iraq 

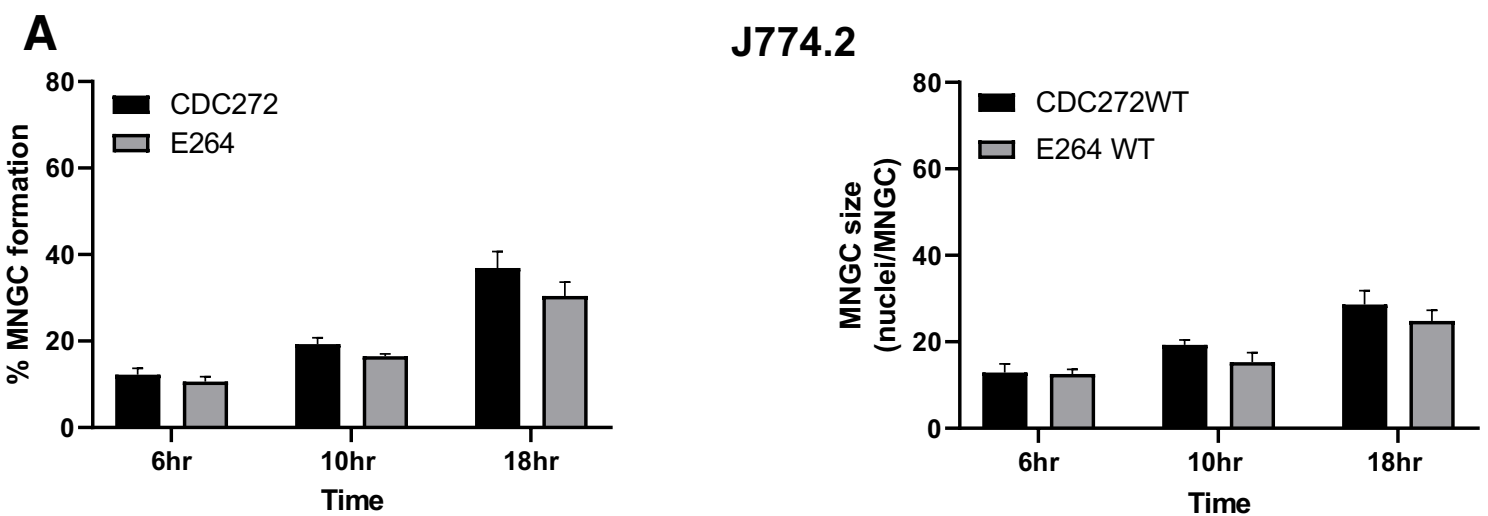

RAW264.7
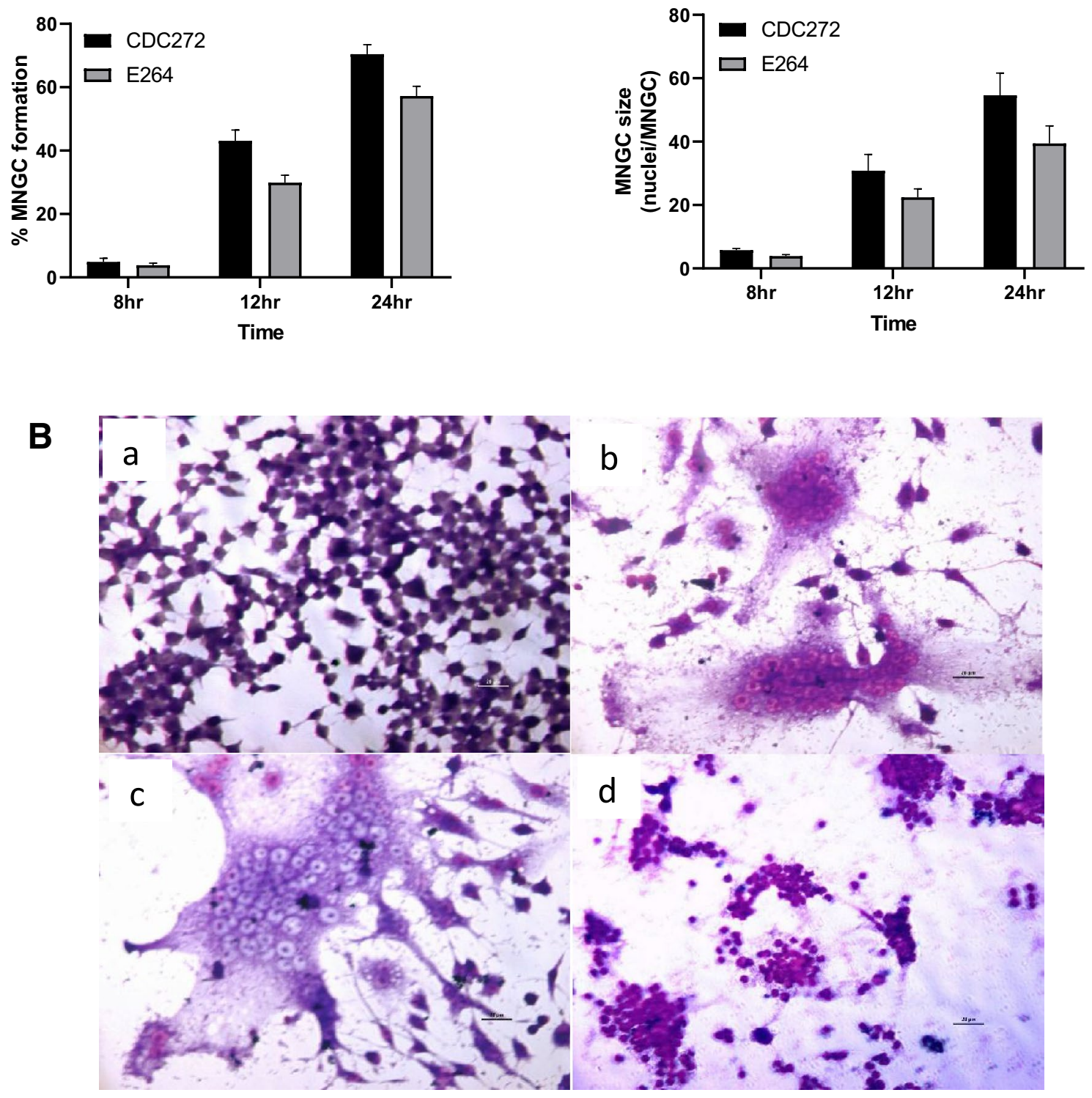
४Fig. 1 MNGC formation induced by B. thailandensis strains E264 and CDC272 in J774.2 and RAW264.7 mouse macrophages. A Time course of MNGC formation in J774.2 (upper panel) and RAW264.7 (lower panel) mouse macrophages induced by B. thailandensis strains E264 and CDC272. Cells were stained with Giemsa and the number of MNGC (cells with $>3$ nuclei) was determined visually per field of view and expressed as a percentage of the total number of macrophages. The average size of MNGC was also calculated by determining the number of nuclei per MNGC per field of view. The error bars represent the standard error of the mean from three independent experiments, each comprising ten fields of view B. Representative images of Giemsa stained uninfected RAW264.7 cells (a) cells infected with B. thailandensis CDC272 (b) or E264 (c) for $16 \mathrm{~h}$ and with CDC272 for $24 \mathrm{~h}$ showing cell necrosis (d). Images were captured using by light microscopy using the $40 \times$ objective, scale bar $20 \mu \mathrm{m}$

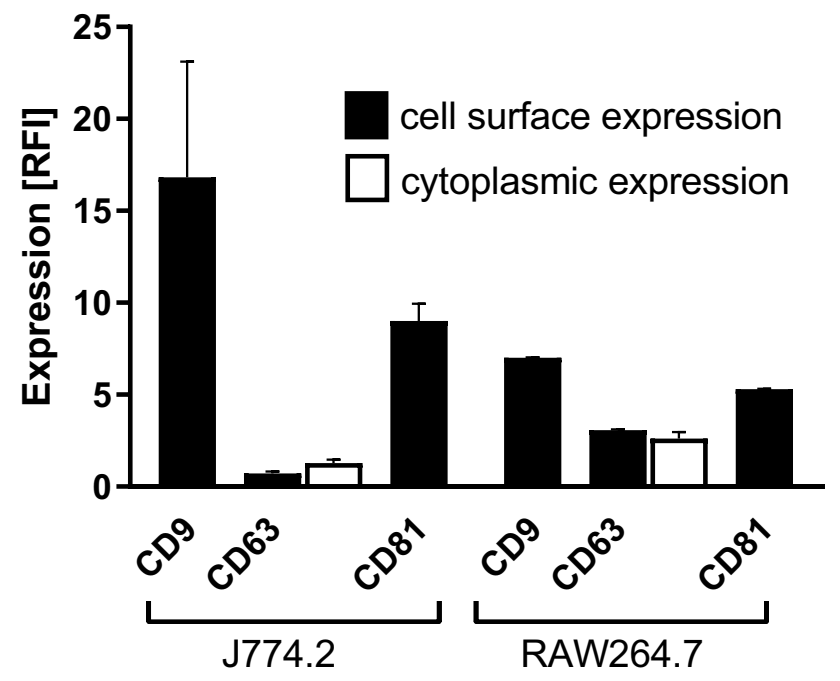

Fig. 2 Expression of CD9, CD63 and CD81 tetraspanins by J774.2 and RAW264.7 mouse macrophages. The relative levels of expression were measured by flow cytometry and are expressed as Relative Fluorescence Intensity (RFI) i.e. MFI of anti-tetraspanin antibody/ MFI isotype control. The data represent at least two experiments performed in duplicate. The data are for live (unfixed) cells except for data shown for CD63 (white fill) which depicts cytoplasmic staining determined using fixed and permeabilised cells 

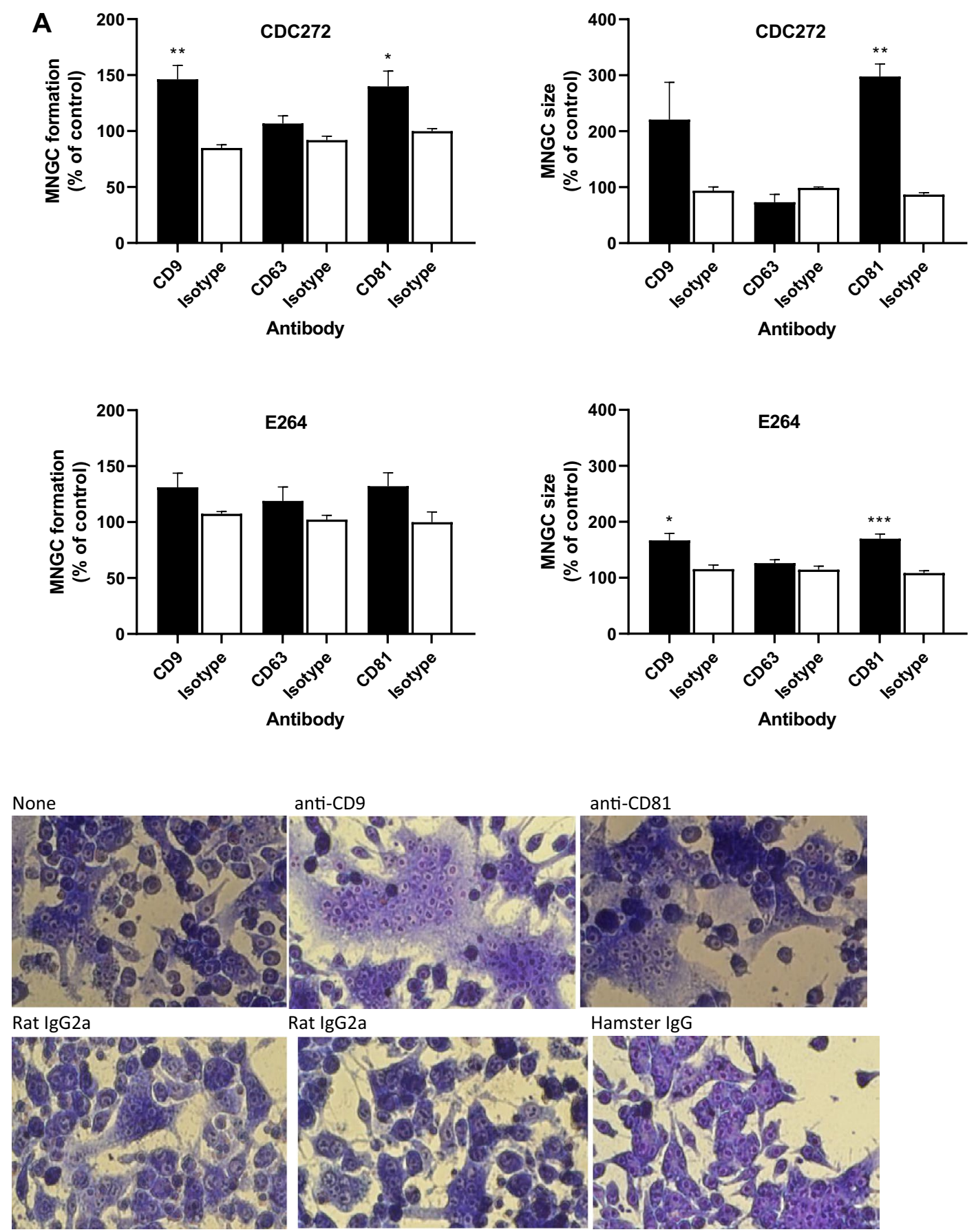

Fig. 3 Effect of mAb to CD9, CD63 and CD81 on MNGC formation induced by B. thailandensis strains CDC272 and E264 in J774.2 A and RAW264.7 macrophages B. Macrophages were pre-treated with test $\mathrm{mAb}$ or isotype-matched controls for $1 \mathrm{~h}$ prior to infection with bacteria and MNGC formation was assessed as described previously. The data are presented as a percentages of the MNGC formed (\% of MNGC) (left panel) or average MNGC size (right panel) compared to the untreated (no antibody) control. The results are representative of at least three independent experiments, with error bars showing standard error of the mean. Statistical analysis was carried out between the test $\mathrm{mAb}$ (black fill) and the matched isotype control (white fill) using Welch's $t$ test. ${ }^{*} p<0.05$, ${ }^{*} p<0.001$,
$* * * p<0.0001$. Pairs with no asterisk showed no significant difference. Representative images of untreated, anti-CD9, anti-CD81 and isotype control-treated J774.2 cells infected with CDC272 and fixed and stained after $16 \mathrm{~h}$ are shown in the lower panel of $\mathbf{B}$. $\mathbf{C}$ shows the effect of $\mathrm{mAb}$ to the tetraspanins (black fill) and corresponding isotype control (white fill) on B. thailandensis infection. J774.2 cells were pre-incubated with antibodies as described above prior to infection $(2 \mathrm{~h})$ and after kanamycin protection to remove extracellular bacteria $(2 \mathrm{~h})$, macrophages were lysed and the number of surviving intracellular bacteria determined (cfu/10,000 host cells). Data were analysed as for $\mathbf{A}$ and $\mathbf{B}$, but no significant differences were found between anti-tetraspanin $\mathrm{mAb}$ and the isotype control-treated samples 

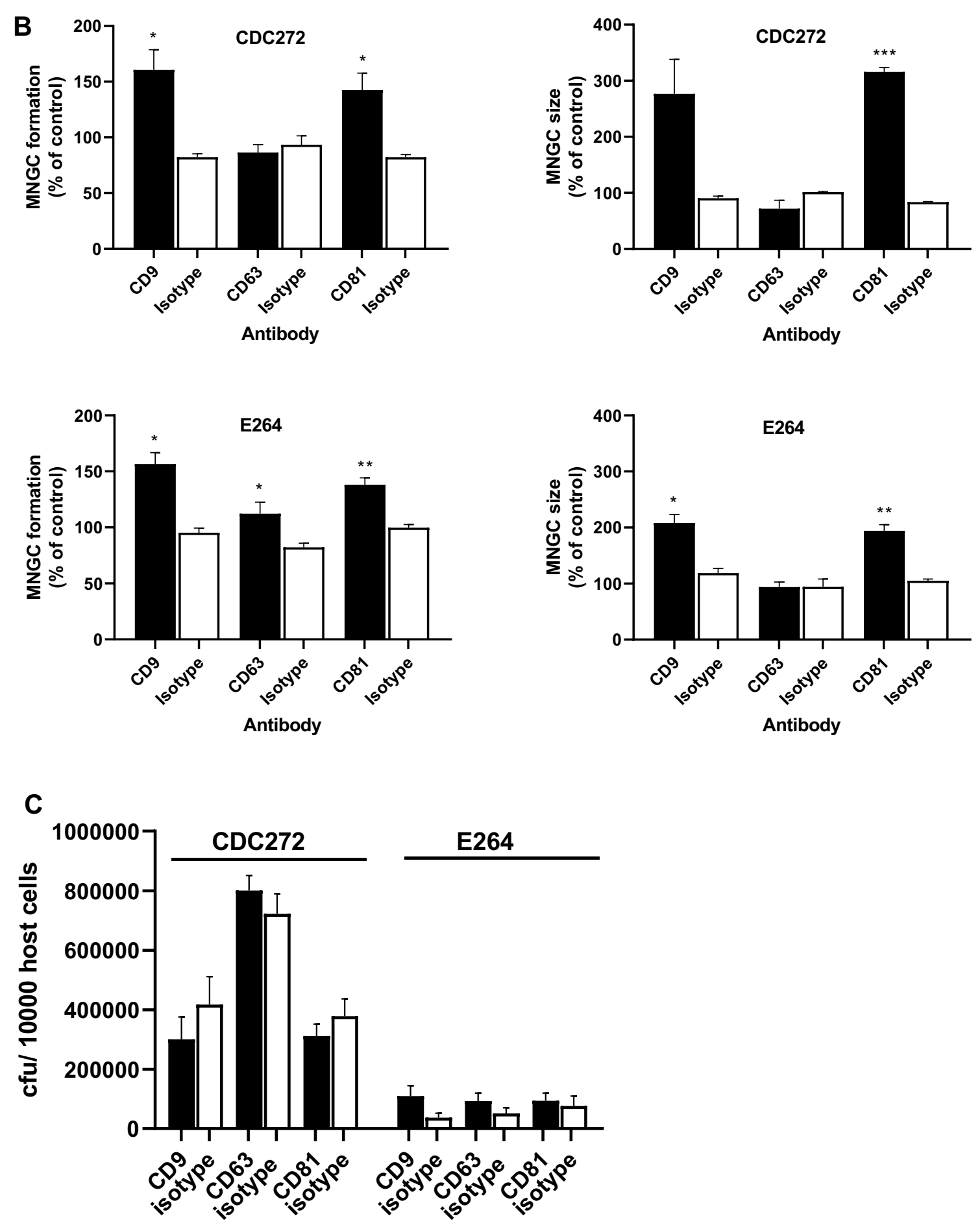

Fig. 3 (continued) 

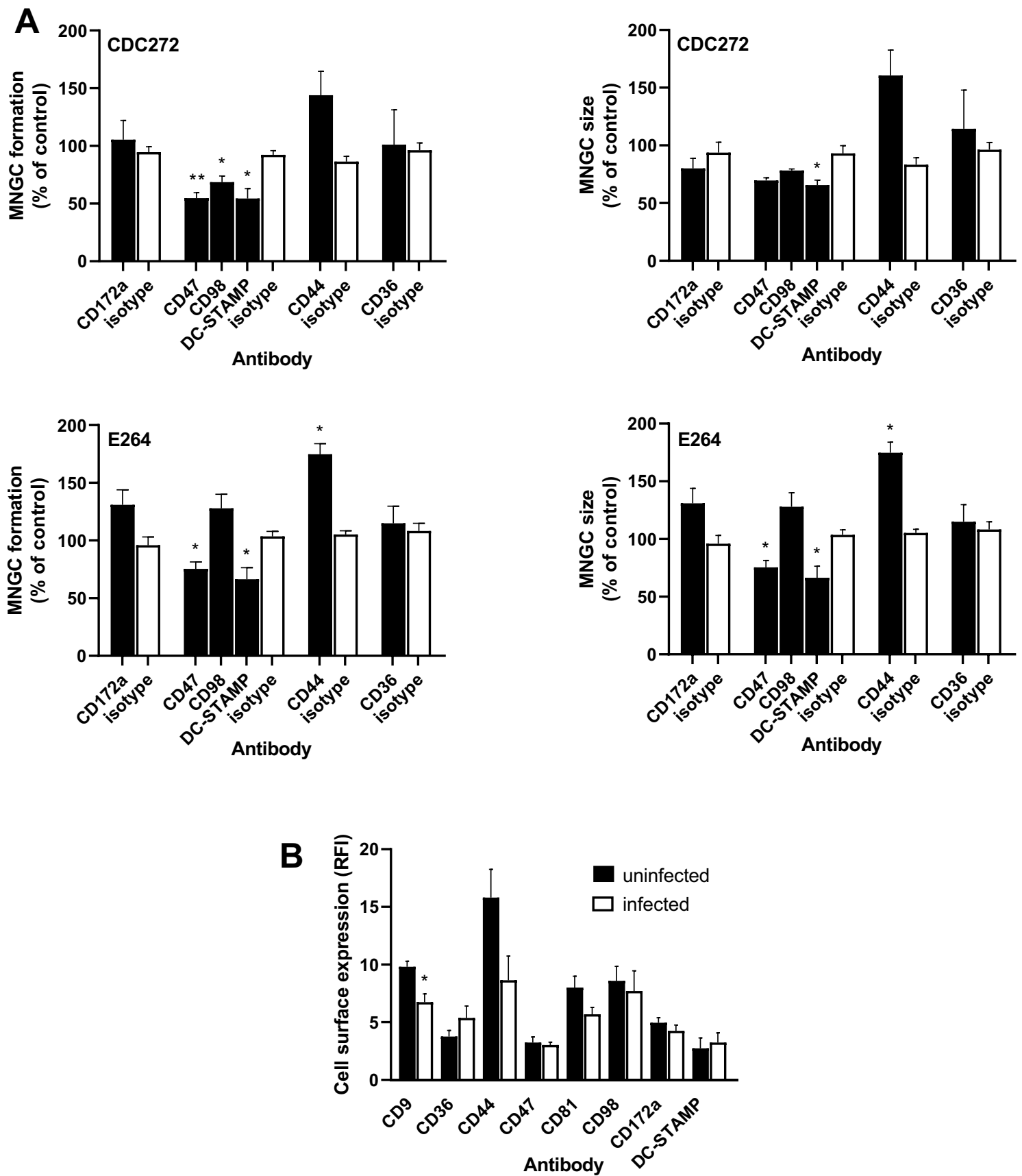

Fig. 4 Effect of non-tetraspanin antibodies on B. thailandensis induced MNGC formation in J774.2 macrophages. A Macrophages were pre-treated with test mAb or isotype-matched control for $1 \mathrm{~h}$ prior to infection with bacteria and MNGC formation was assessed as described previously. The data are presented as percentages of MNGC formed (\% MNGC) (left panel) or average MNGC size (right panel) compared to the untreated (no antibody) control. The results are representative of at least three independent experiments, with error bars showing standard error of the mean. Statistical analysis was carried out between the test mAb (black fill) and the matched isotype control (white fill) using Welch's $t$ test. $* p<0.05$, ** $p<0.001$. Pairs with no asterisk showed no significant difference. B Changes in cell surface expression of antigens $3 \mathrm{~h}$ after infection of J774.2 cells with B. thailandensis $\mathrm{CDC} 272$ was assessed by flow cytometry relative to uninfected controls at the same point. The data represent RFI values from at least four independent experiments performed in duplicate. Statistical analysis was carried out between the uninfected (black fill) and infected (white fill) cells using Welch's $t$ test. ${ }^{*} p<0.05$. Pairs with no asterisk showed no significant difference 

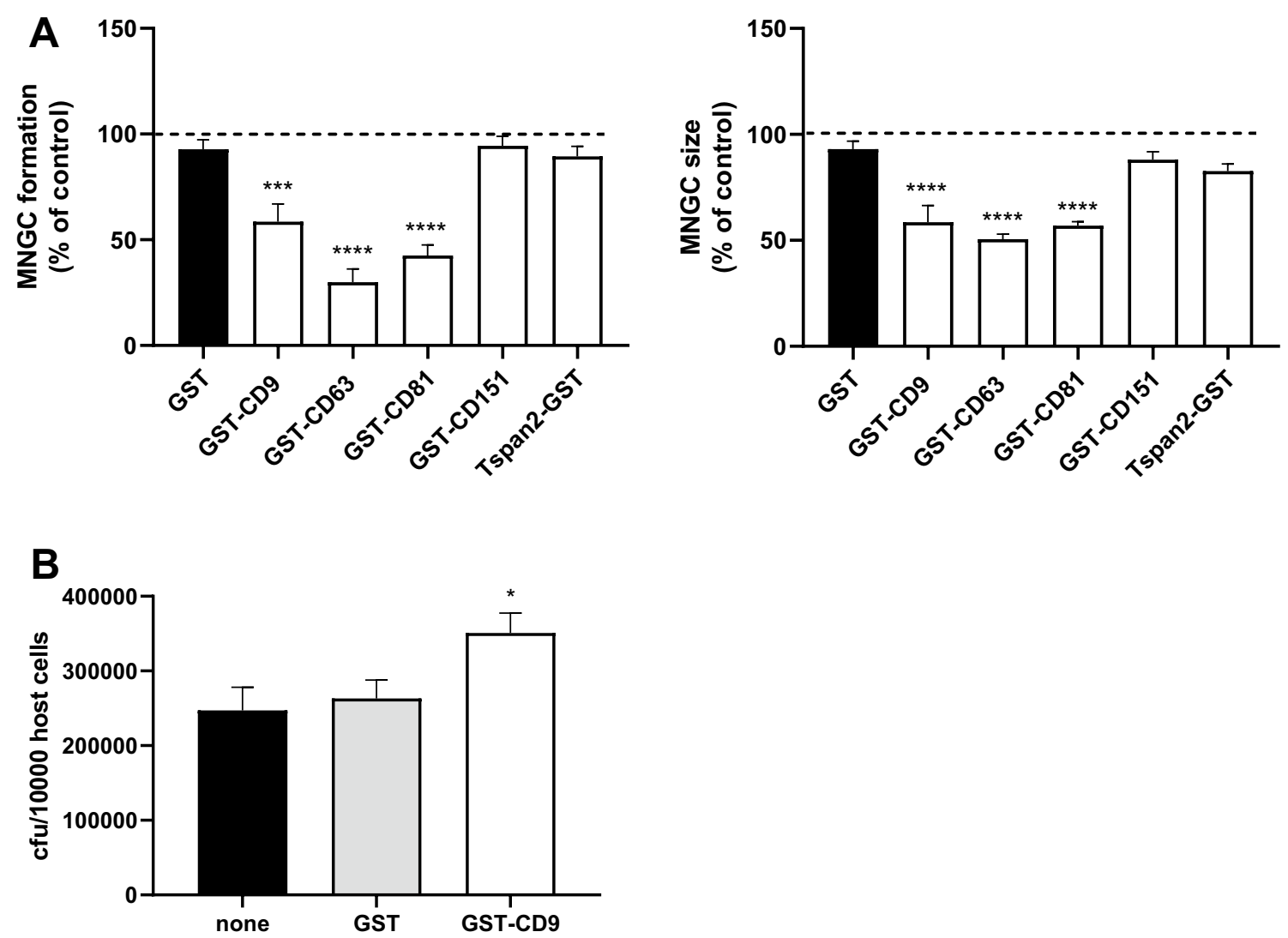

Fig. 5 Effect of recombinant GST-EC2 on MNGC formation induced by $B$. thailandensis strains CDC272 and E264 in J774.2 macrophages. A Macrophages were pre-treated with GST or GST-EC2 proteins for $1 \mathrm{~h}$ prior to infection with bacteria and MNGC formation was assessed as described previously. The data are presented as the percentages of MNGC formed (\% MNGC) (left panel) or average MNGC size (right panel) compared to the untreated (no recombinant protein added) control. The results are representative of at least three independent experiments, with error bars showing standard error of

the mean. Significance of difference from GST control was established by One Way ANOVA with Dunnett's post-test. *** $p<0.001$; $* * * * p<0.0001$. B Shows the effect of GST proteins on B. thailandensis infection. J774.2 cells were pre-incubated with or without the recombinant proteins prior to infection $(2 \mathrm{~h})$ and after kanamycin protection to remove extracellular bacteria $(2 \mathrm{~h})$, macrophages were lysed and the number of surviving intracellular bacteria determined (cfu/10,000 host cells). Significance of difference from GST control was established as described for $\mathbf{A}$ 
A

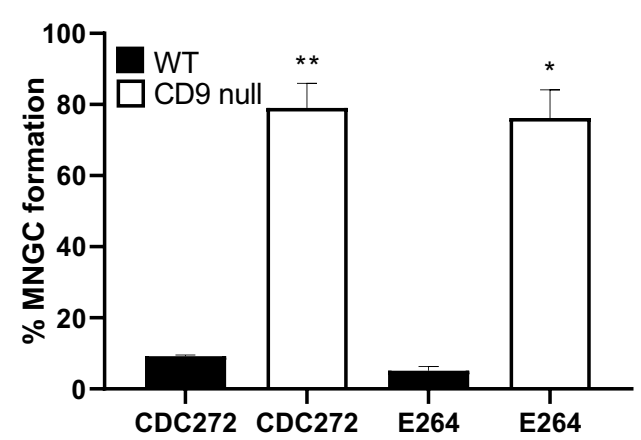

B

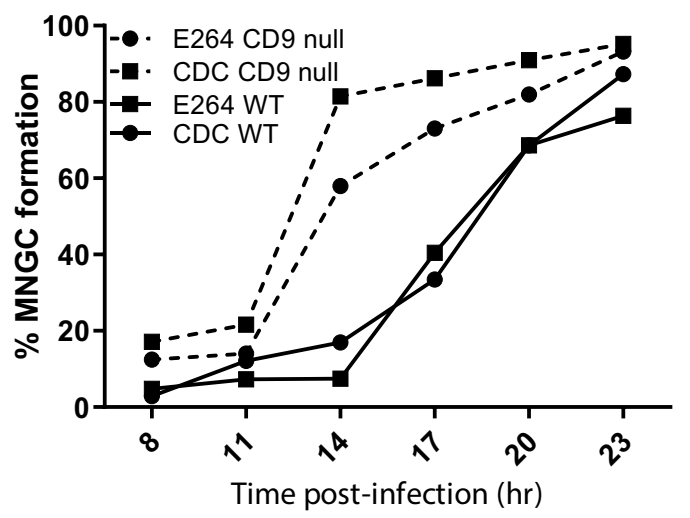

C

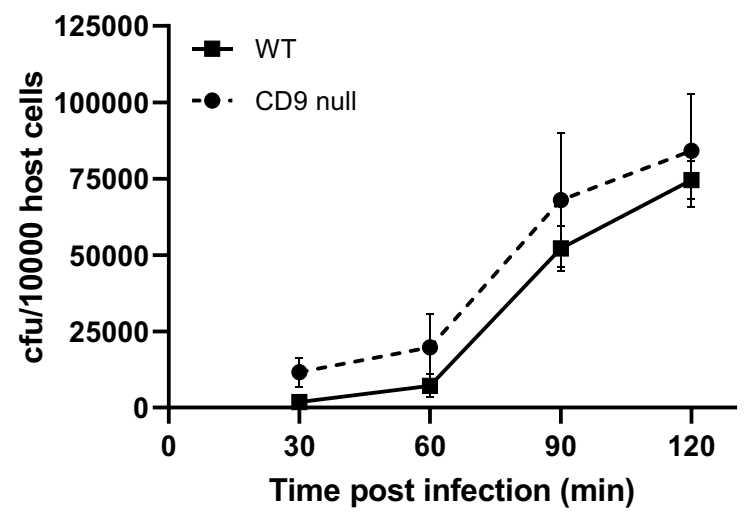

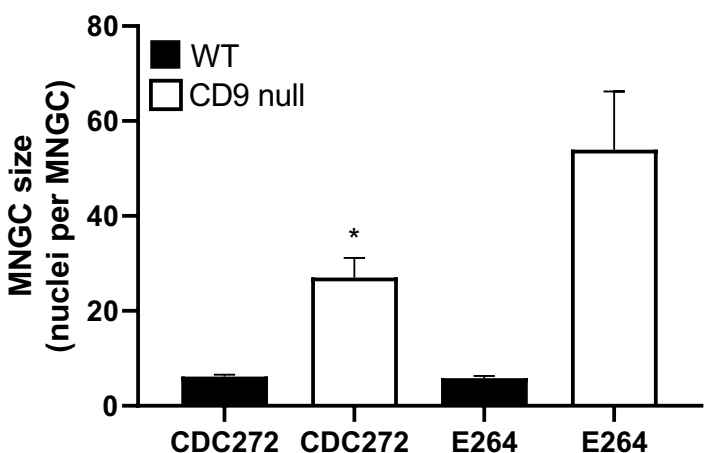

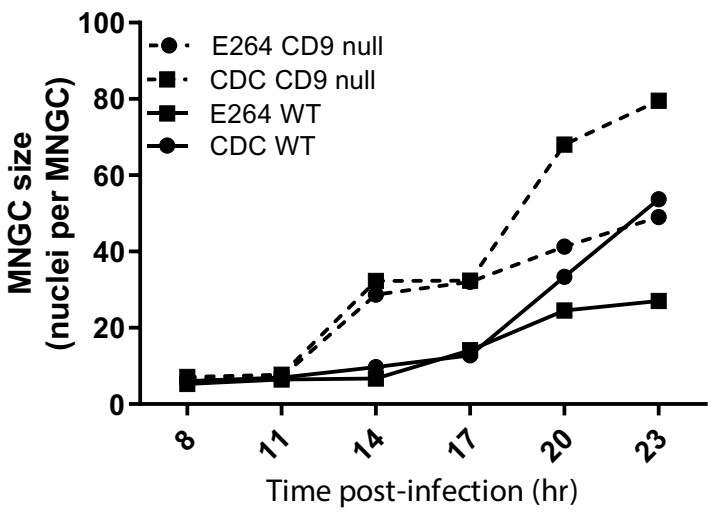

D

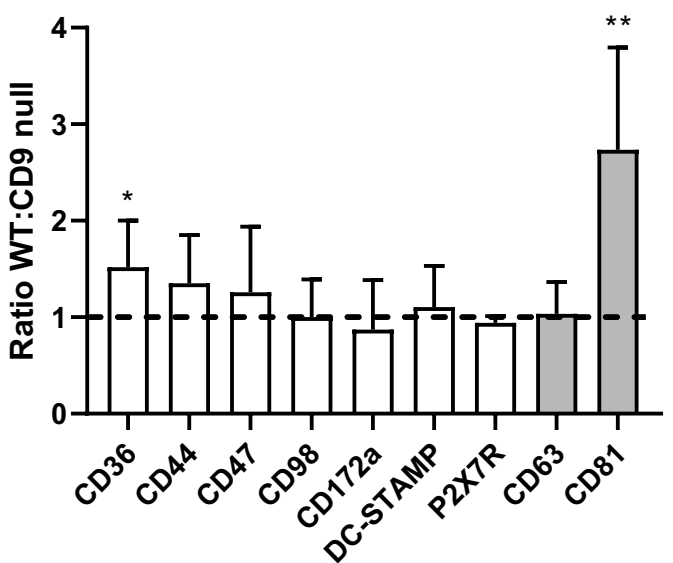

Antibody
Fig. 6 Effect of CD9 knock-out on MNGC formation induced by $B$. thailandensis strains CDC272 and E264 in mouse macrophages. A Following infection of macrophages derived from WT (black fill) or CD9 null mice (white fill) with both strains of bacteria, MNGC formation was assessed after $14 \mathrm{~h}$ as described previously. The results are representative of three independent experiments, with error bars showing standard error of the mean. Statistical analysis was carried out between the WT and KO macrophages using Welch's $t$ test. ${ }^{*} p<0.05, * * p<0.001$. B Time course analysis shows that MNGC formation occurs earlier and to a greater extent in CD9 null mouse macrophages. C Analysis of early infection of WT and CD9 null mouse macrophages by $B$. thaillandensis CDC272 was performed after the kanamycin protection assay by lysing the macrophages at various time points and determining the number of surviving intracellular bacteria determined (cfu/10,000 host cells). D The surface expression of tetraspanins CD63 and CD81 (grey fill) and non-tetraspanin fusion-related proteins (white fill) was assessed by flow cytometry. RFI values for WT and CD9 null are shown as a ratio, with error bars showing standard error of the mean from at least three independent experiments performed in duplicate. Statistical analysis was assessed by one sample $t$ test, ${ }^{*} p<0.05, * * p<0.001$ 

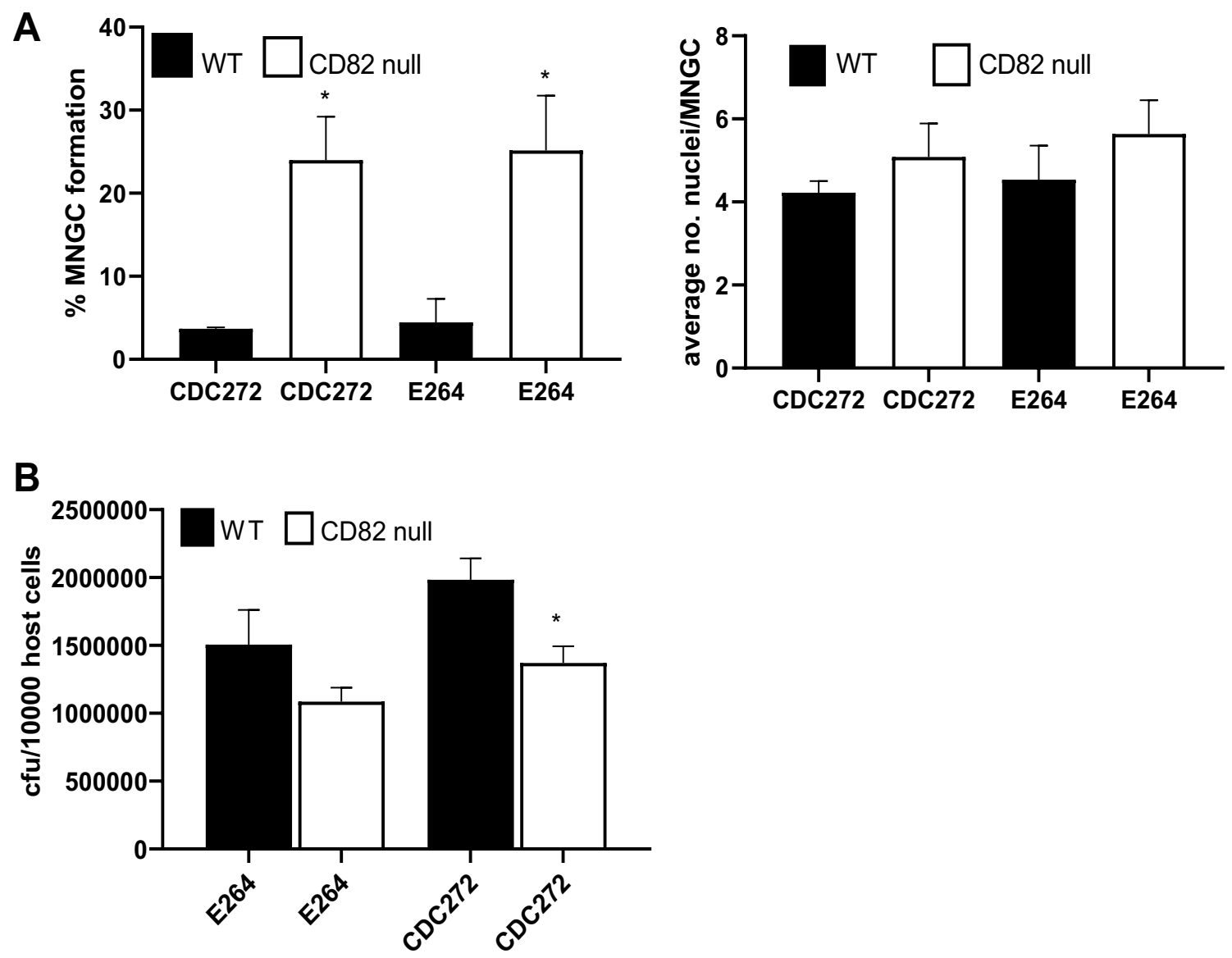

Fig. 7 Effect of CD82 knock-out on MNGC formation induced by $B$. thailandensis strains CDC272 and E264 in mouse macrophages. A Following infection of macrophages derived from WT (black fill) or CD82 null mice (white fill) with both strains of bacteria, MNGC formation was assessed after $14 \mathrm{~h}$, as described previously. The results are representative of three independent experiments, with error bars showing standard deviation of the mean. Statistical analysis was carried out between the WT and KO macrophages using Welch's $t$ test.
$* p<0.05, * * p<0.001$. B Analysis of infection of WT and CD82 null mouse macrophages by $B$. thaillandensis CDC272 was performed after the kanamycin protection assay by lysing the macrophages $2 \mathrm{~h}$ and determining the number of surviving intracellular bacteria determined (cfu/10,000 host cells). Statistical analysis was carried out between the WT (black fill) and CD82 null (white fill) cells using Welch's $t$ test. ${ }^{*} p<0.05$. Pairs with no asterisk showed no significant difference 

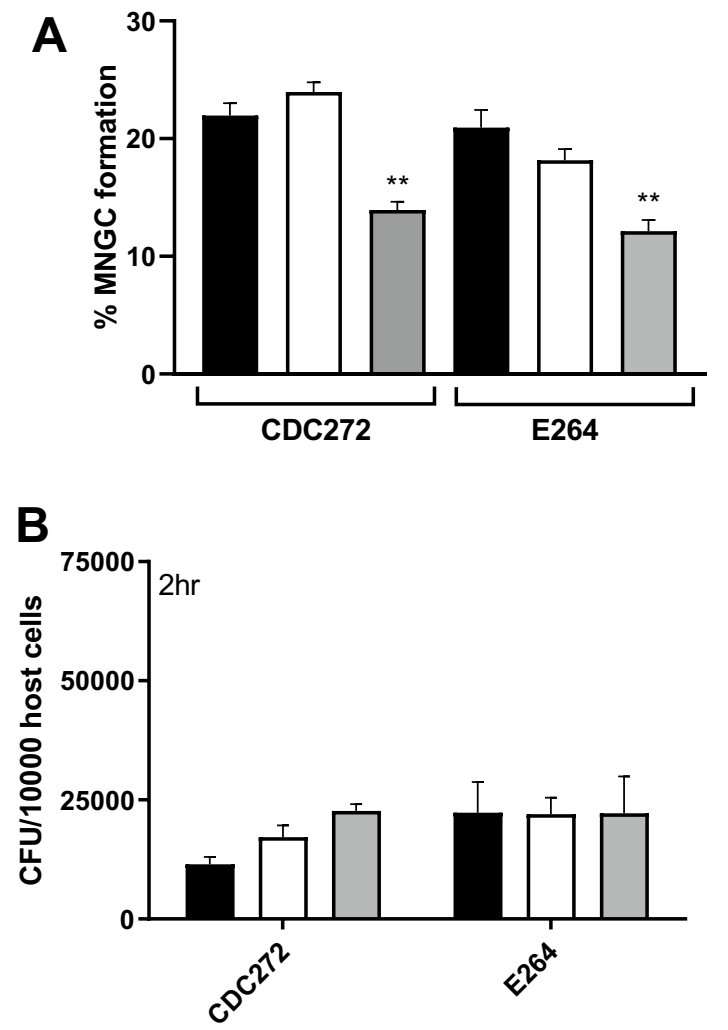

Fig. 8 Effect of CD9 overexpression on MNGC formation induced by B. thailandensis strains CDC272 and E264 in mouse macrophages. A The capacity of $B$. thailandensis strains E264 and CDC272 to induce MNGC formation in WT J774.2 cells (black fill), J774.2 cells stably transfected to overexpress GFP (white fill) or J774.2 cells stably transfected to overexpress mouse CD9-GFP (grey fill) was assessed $16 \mathrm{~h}$ after infection as described previously. Data represent three independent experiments, with error bars showing standard error of the mean. Statistical differences between the cell types was assessed
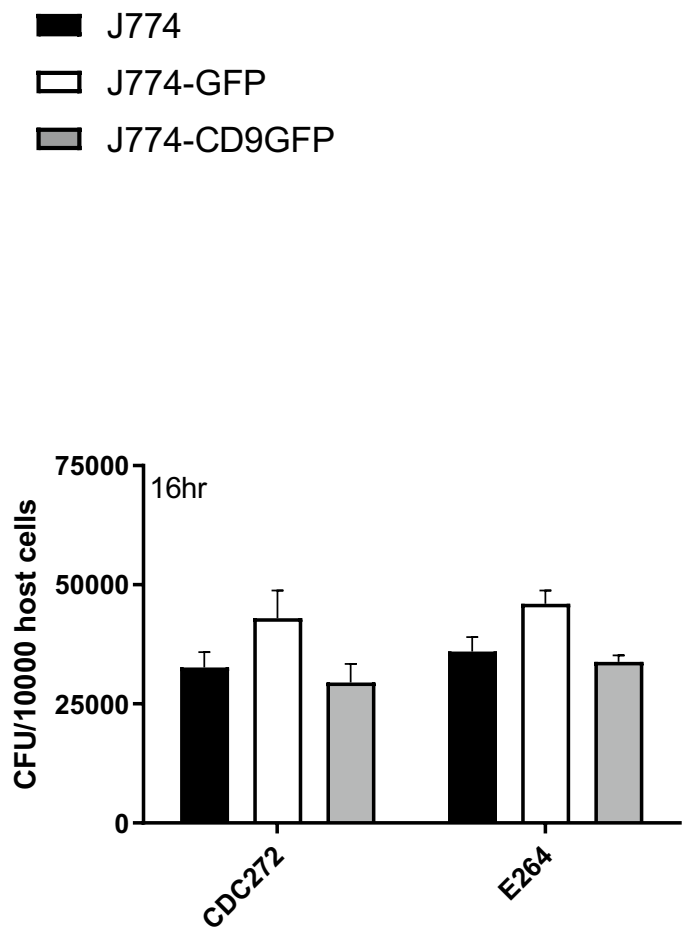

by two way ANOVA, $* * p<0.001, n s$ non-significant. B Survival of B. thailandensis strains E264 and CDC272 in WT J774.2 cells (black fill), J774.2 cells stably transfected to overexpress GFP (white fill) or J774.2 cells stably transfected to overexpress mouse CD9-GFP (grey fill). Bacterial survival was assessed at $2 \mathrm{~h}$ and $16 \mathrm{~h}$ after infection by lysing the cells and determining the number of live intracellular bacteria as described previously. The data was analysed as for $\mathbf{A}$, but no statistical differences between the WT or transfected J774.2 cells were found 